\title{
Using 2D and 3D Computer Games \\ to Detect Colorblindness - a Comparative Study
}

\author{
Maciej Laskowski ${ }^{1}$ \\ 1 Institute of Computer Science, Lublin University of Technology, Poland
}

\begin{abstract}
Computer games have accompanied the development of computer technologies since the very beginning. Despite their basic, purely entertainmenttargeted appliance, games can also be used for many other purposes. Medical applications are especially interesting, as games (especially different kinds of simulations) are widely used for training personnel, e.g. to perform certain procedures or in learning to use equipment. This allows the trainees to gain knowledge and proper habits, as well as test themselves in different situations without any risk. Computer games can also be used as a diagnostic tool, although this topic is still insufficiently researched. This paper discusses the possibility of using serious games for diagnosing color vision disorders, focusing especially on two problems: differences in diagnosing colorblindness using 2D and 3D environments, and the influence of individual features, such as reflex or agility, on the diagnostic process.
\end{abstract}

\section{Color Vision Disorders - a Short Overview}

According to popular opinion, colorblindness is the inability to either perceive colors at all or distinguish between red and green. This is not completely true, as - generally speaking - having a color vision disorder (CVD) means that a person is unable (or has reduced ability) to tell certain colors or their shades apart under normal lightning conditions (Shevell, 2003).

Depending on the disorder type, a person may either not be able to perceive colors at all (total color blindness), or may have a significantly reduced visible color spectrum (in the case of dichromacy, where one type of retinal cones is missing or strongly underdeveloped), or may not be able to distinguish between certain shades of colors (in the case of anomalous trichromacy, where one type of retinal cones is slightly underdeveloped or not working properly) (Shevell, 2003). 
Total color blindness is an extremely rare condition, affecting from about $0.00001 \%$ (cone monochromacy) up to $0.001 \%$ (rod monochromacy) of the total human population (McIntyre, 2002). Due to this, it will not be investigated further in this paper.

In the retina, there are three types of cone cells, which are receptive to different wavelengths of light: in the most basic (and simplified) way we may say that one is sensitive mainly to reds, another to greens and another to blues (Figure 1).

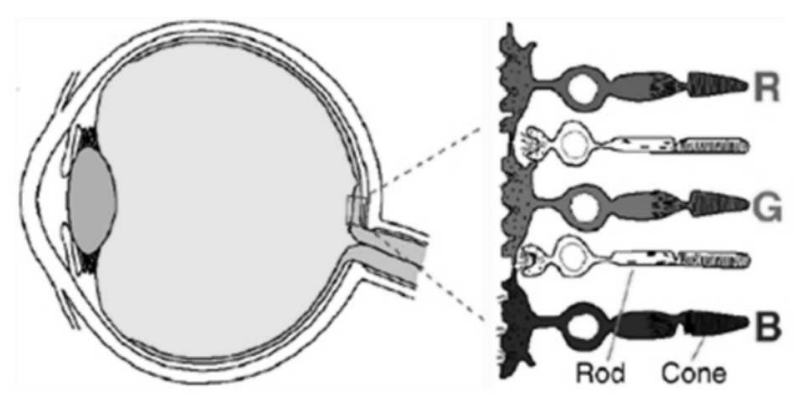

Figure 1. Photosensitive cones in the human retina (source: Mushaboomde signcom, 2015)

Both dichromacy and anomalous trichromacy are divided into three subtypes (respectively: protanopia, deuteranopia and tritanopia in the case of dichromacy, and protanomalia, deuteranomalia and tritanomalia in the case of anomalous trichromacy), depending on the type of retinal cones missing or not working properly.

It is estimated that about $8-10 \%$ of the whole human population is affected with color vision disorders. Based on this estimation, a Community Interest Company called Colour Blind Awareness runs a campaign called \#1ineveryclassroom, targeted at informing people that in each classroom there is at least one pupil with color vision disorder (Colourblindawarenessorg, 2015).

Although relatively high frequency, it must be noted that colorblindness is not distributed evenly among the human population - it affects more men than women, except for monochromacy, tritanopia and tritanomaly, the last two caused by a mutation carried on gender-independent chromosome 7 (McIntyre, 2002).

Moreover, color vision disorders more often affect people of European ancestry. It is estimated that - considering the male population only - one in twelve Caucasians is colorblind, compared to one in twenty Asians and one in twenty-five Africans (Masataka et al., 2008). 


\section{Colorblindness and Life of the Affected Persons}

The problem of impact of color vision disorders on the professional lives of affected persons (especially in the logistics sector but also e.g. for medical diagnosticians or chemists) has been widely described and discussed in the literature (e.g. Campbell et al., 2004; Spalding, 2004; Steward et al., 1989).

Problems concerning the everyday life of the colorblind person are less analyzed, although they span from being unable to determine the ripeness of fruit or to read a colored chart up to having difficulties recognizing proper medicine.

In each of these cases, being aware of having a color vision disorder is pretty important, as colorblindness has a negative influence on quality of life or - in some cases - even endangers the personal safety of the person affected.

\section{Using Computer Games to Detect Colorblindness}

There are various different methods for detecting colorblindness, with pseudoisochromatic Ishihara plates being the most commonly used, mainly due to their relative reliability, test simplicity and low cost (Yates et al., 2001).

Other types of detection methods include (French et al., 2008; Shevell, 2003; Yates et al., 2001): arrangement methods (where the tested person is asked either to select set elements with colors matching or close to the ones given by the examiner or to arrange the set elements in a certain order), lamp methods (where examinees are just tested for their abilities to tell the colors used in navigation apart), and spectral methods (where the tested person has to obtain a certain color by mixing two color lights in a certain ratio).

Each of those methods has its own accuracy (which may lower over time, due to e.g. equipment wearing out), cost per examination, and limitations, e.g. minimum age of the examinee.

Moreover, only the pseudoisochromatic plates (with top accuracy estimated as $90-95 \%$ in the first 2 years of use) are considered as usable for screening tests, where a large number of persons have to be tested over a relatively short period of time. Test circumstances (e.g. rush) may have a negative impact on quality. Research (Laskowski, 2015) shows that in the case of colorblindness, detection in up to $1 / 3$ of patients is not informed by the characteristics (or even type!) of the owned color vision disorder. 
The problem of ensuring diagnostic accuracy is especially important in countries where being diagnosed as colorblind may result in exclusion from certain occupations (e.g. as driver or pilot) or may even prevent one from having a non-professional driving license (French et al., 2008). It is also worth noticing that in some cases people tend to pretend to have a color vision disorder, as this would allow them to be transferred to a different position - e.g. on Indian railways, the colorblind are not allowed to work as motormen but are shifted to be conductors, which usually means extra income (French et al., 2008).

This proves that, in order to provide a reliable tool for screening tests, there is a need to develop a new, automatized method for detecting colorblindness. This method should have the following features:

- It should be low cost, both in implementation and use.

- It should be accessible to and understandable for anyone, regardless of age, culture and language spoken.

- Test results should be evaluated automatically as quickly as possible, in order to speed up the screening process and to minimize the possibility of error.

- Each detected color vision disorder should be corroborated by an expert, which would fulfill the requirement of minimizing the number of patients to be tested by a human.

The idea of using simple computer games for detecting color vision disorders was first postulated in a paper by Bober et al. (2011). Despite the fact that the primary method was focused only on detecting colorblindness in candidates for drivers and was limited to the player deciding whether to cross the intersection or to wait for a light to change (based on randomly ordered traffic lights), the obtained results were quite successful - four potential red-green dichromates were detected in a population of 80 experiment participants.

This experiment - although simple and requiring further, more planned development, especially concerning proper color display - proved that computer games may serve as a tool for detecting colorblindness.

This observation served as the basis for the second experiment, aimed at detecting various types of colorblindness, not limited only to certain kinds of dichromacy. Players were asked to choose one of two colors displayed on the screen at the same time in order to match the model color. The participants were presented with 125 color pairs in total, and were given 5 seconds to make each choice. The game used seven different color sets: one neutral (where colors could be told apart by anyone, regardless of the correctness of their color vision), three for each type of dichromacy and three for each 
type of anomalous trichromacy. Moreover, sets were complementary in corresponding types of color vision disorders: e.g. people with protanomaly should be able to distinguish colors that are perceived wrongly by people with protanopia.

In order to develop the discussed color sets, so-called "confusion axes" were used - intersecting lines (for each type of dichromacy) going through CIE 1931 color space (see Figure 2 for details). Colors placed on those lines are hard (or in the case of severe color vision disorders - impossible) for people with certain types of dichromacy to distinguish (Yates et al., 2001).
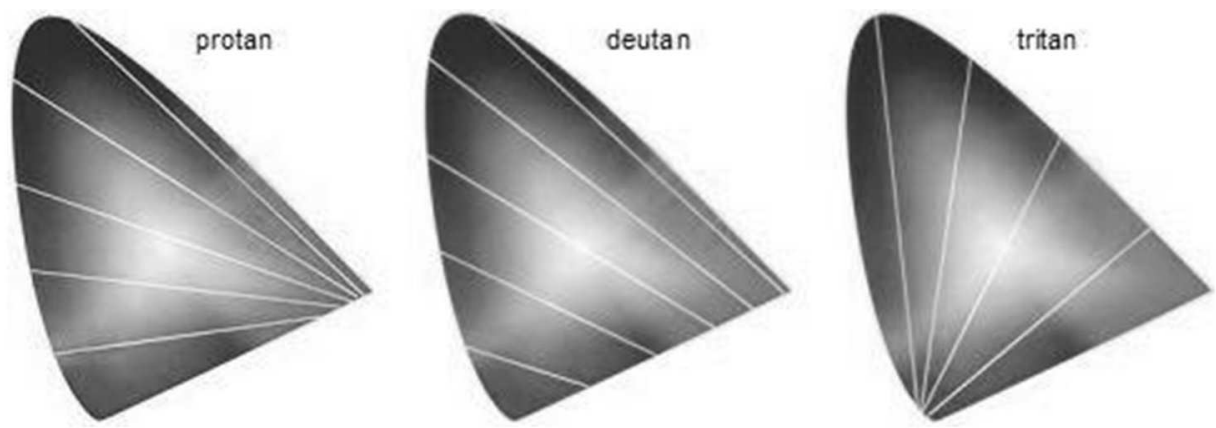

Figure 2. Schematic representation of the CIE color triangle with confusion lines for protan, deutan, and tritan defects (source: Opticaldiagnosti cscom, 2015)

Table 1 shows samples from the color set used to detect protanomaly. Colors are presented using the RGB color model, where each color is described an RGB triplet $(\mathrm{r}, \mathrm{g}, \mathrm{b})$ - each pair of characters representing hexadecimal values varying from 0 up to $\mathrm{FF}$ (from 0 to 255 in the decimal system).

Table 1. Sample color pairs from set used to detect protanomaly

\begin{tabular}{|c|c|}
\hline Color 1 RGB value & Color 2 RGB value \\
\hline \#344C51 & \#704E53 \\
\#F1EC00 & \#B5FD03 \\
\#529C6B & \#B19D6D \\
\hline
\end{tabular}

Source: Own work based on DeMarco et al. (1992), Derefeldt et al. (1995), Machado et al. (2009)

Proper color representation is one of the most puzzling problems concerning computer graphics. Depending on the graphical and display hardware, the presented colors may differ noticeably. Usually those differences 


\section{Maciej Laskowski}

are corrected by a Color Management System implemented into the OS, although some applications have their own CMSs. The described game was created using HTML5 and JavaScript, in order to use the Color Management Systems implemented in the web browsers.

The whole experiment was divided into four phases: the first one the introductory phase - consisted of 6 color pairs using a neutral color scheme. This stage was used mainly to introduce the player to the game. The second phase, consisting of a total of 84 pairs, used the following pattern: pairs for each type of anomalous trichromacy were separated by pairs of neutral colors. Upon completion of this stage, the colors selected by the player were analyzed (using the algorithm presented below) in order to choose the potential color vision disorder, based on the number of wrong choices.

The analysis algorithm was relatively simple: the results were sorted in descending order and stored in a two-dimensional array (CVD type and number of errors). Errors referring to neutral color schemes were ignored in the analysis. If the overall number of errors exceeded 63 (half of all possible errors), the analysis was not performed and the user was treated as if no color vision disorder was detected.

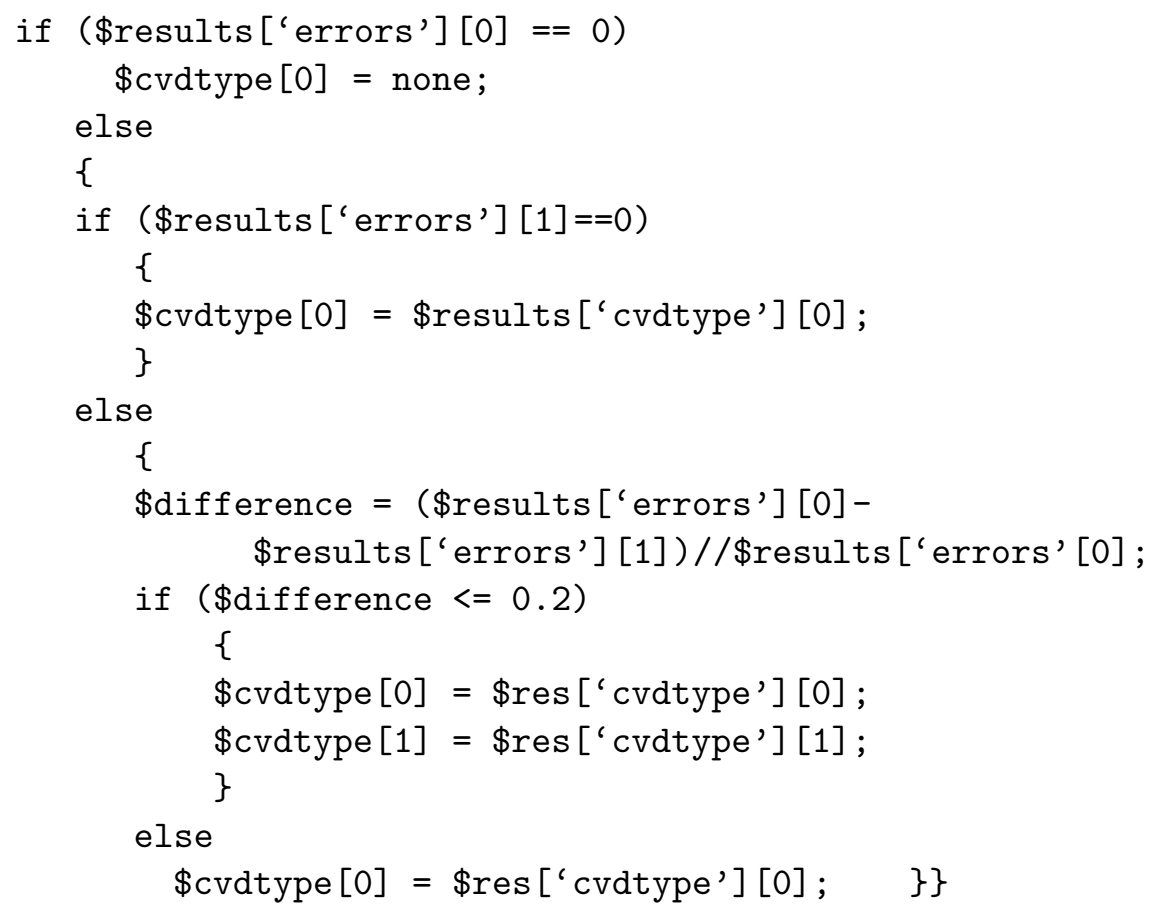


The result of this analysis was used as the basis for the third - precision - stage, which consisted of 30 pairs. This phase was used - if at least one potential color vision disorder was detected - to determine if the tested person was affected with dichromacy or anomalous trichromacy. The color schemes based on this analysis were used alternately with neutral color schemes. If the analysis detected no potential color vision disorders or results from the previous phase exceeded the tolerance scale, the color schemes at this stage were used in the same manner as in the previous one.

After completion of the third phase, the analytic algorithm was used again in order to select the potential color vision disorder, based on the highest error ratio.

The fourth stage was used in order to confirm the diagnosis, as it used all the color schemes from the second stage supplemented by the scheme chosen based on the diagnosis. This phase consisted of 5 color pairs. The final analysis was conducted upon completion of the fourth phase.

As the described method is designed to support screening tests, all of the diagnosed cases of color vision disorders are to be tested offline using Ishihara pseudoisochromatic plates (as a widely used method in medical examination rooms) in order to corroborate the diagnosis made by the system.

The method's effectiveness was corroborated after running tests on a group of 143 persons. Based on raw data (without implementing a tolerance margin), it allowed the number of people requiring medical examination for color vision disorders to be decreased by almost 3/4. After implementing a tolerance margin - in order to eliminate e.g. random mistakes - the number of people requiring medical examination for color vision disorders was reduced by over $90 \%$ (13 out of 143). For further details, including a complete description of this experiment and analysis of its results see: Laskowski (2015).

\section{Research Problems}

The discussed game did prove the accuracy of the described method, which may serve as a reliable and cheap alternative to methods used currently.

But it should be noticed that the game used in the described experiment was as simple as possible: the user was just asked to choose one of two squares. The application interface was minimalistic (Figure 3) and the position of all elements was static. Time was the only limitation; there was 
a maximum of 5 seconds allotted per pair. Therefore, the influence of such individual features as reflex, perceptivity or agility was minimized.

Moreover, it must be noted that almost all of the currently used color vision disorder detection methods are "static" - they are focused only on either detecting CVDs or testing the ability to recognize certain color signals. This makes conducting research on the influence of those features on diagnosis an interesting problem. Switching from 2D to 3D and its influence on the results may also be another interesting research aspect.

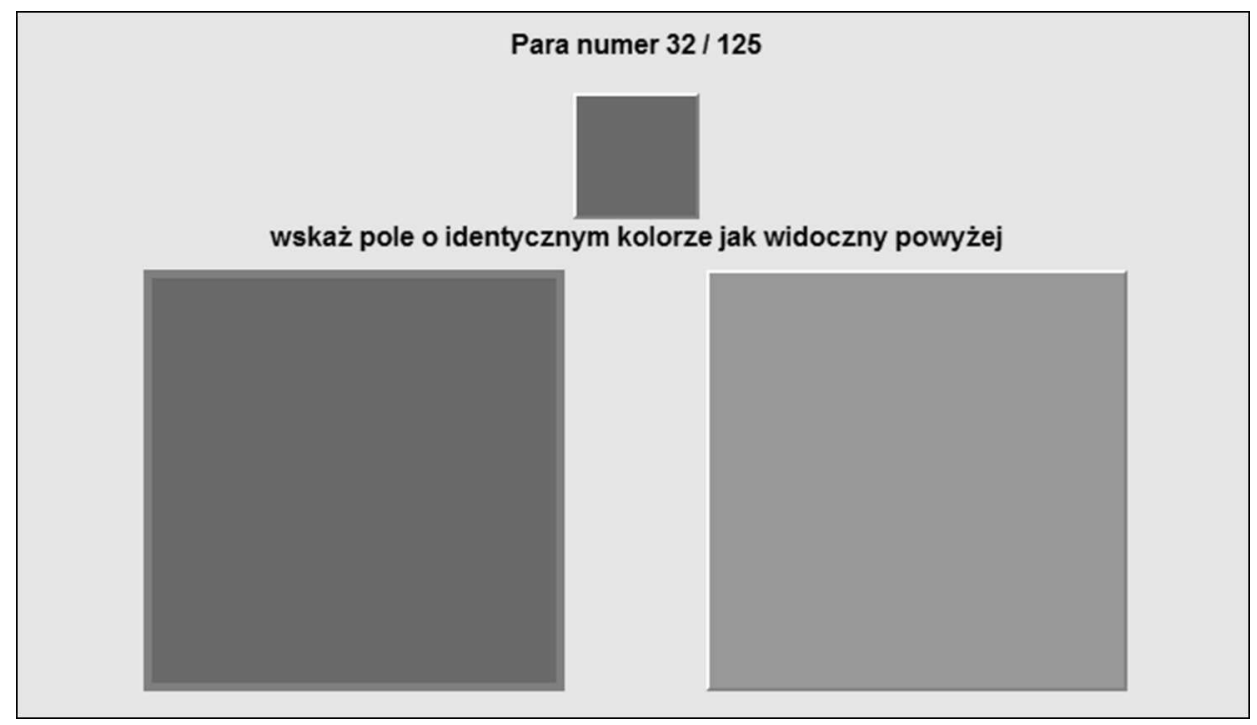

Figure 3. Interface of the model game (source: own work)

In order to answer the questions of how use of a less static game or switching from a $2 \mathrm{D}$ to a $3 \mathrm{D}$ game might influence the results of the previously described experiment, two separate experiments were conducted, based on the algorithm and diagnostic method described above, as well as the same color schemes.

Each experiment engaged two equipotent groups, containing twelve persons each. One group consisted of people who had already been diagnosed with colorblindness, while the second one - consisting of people with normal color vision - served as a control group. It should be noted that people in the first group were not always informed of the specifics (e.g. type) of their diagnosed color vision disorder.

All of the data obtained during those experiments is presented without implementing any error margin. 


\section{Experiment 1 - Influence of Individual Features}

In order to check whether individual features (like agility or reflex) had an influence on the diagnosis made by the system, the author proposed a new color-testing game, based on modified skeet rules.

In order to play the game, the user is asked to shoot one of two color clay pigeons (the one matching the model color, which is presented at the top of the screen). During the whole game, the player is presented with a total 125 pairs of clay pigeons (the same number as in the previous experiment). Each clay pigeon moves across the screen at the same pace and uses the same trajectory, although the starting point at the side of the screen is randomized. The user can shoot just once at each pair and hitting the wrong clay pigeon is considered an error and is subject to analysis. The game interface is presented in Figure 4.

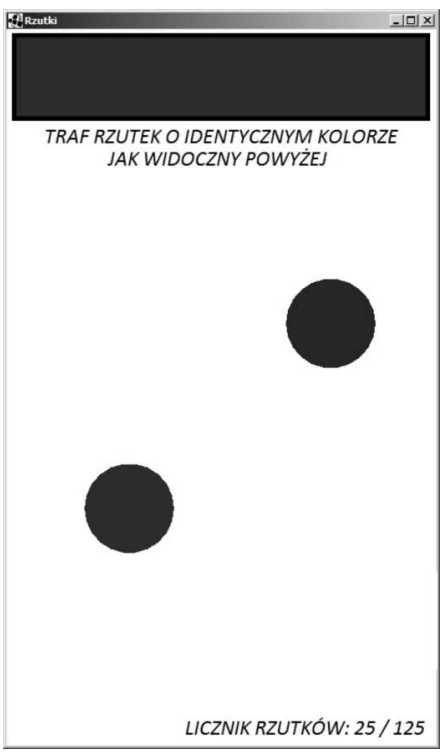

Figure 4. Interface of the ColorSkeet game (source: own work)

\section{Experiment 2 - Diagnosing Potential Color Vision Disorders in a $3 \mathrm{D}$ Environment}

The main problem of creating a game for potential colorblindness detection in 3D is associated with lighting and shading, as those features may add additional shades to the tested color and therefore tamper the color percep- 
tion, which may result in a false diagnosis (especially considering anomalous trichromacy).

To avoid this, the author decided to create a game using "raw" colors on the testing elements. Moreover, the game was set in a dungeon, in order to provide the same lighting conditions for each of the tested elements. The player's goal is to go through a maze (125 rooms). In each room there are two doors, each marked with a color. The player has to decide which door to open, basing their choice on the color in the center of the room (Figure 5). Entering the door with the same color as in the center of the room is considered "right", while entering the door with the other color is interpreted as an error (which is a subject to further analysis for potential colorblindness), although it does not prevent the user from leaving the dungeon - as the game is set to "release" the player from the dungeon after walking through 125 rooms. The 3D environment was used just for the purpose of making the game appealing, as the same diagnostic method was used as in the case of the 2D game.

In order to make the game more entertaining, some rooms require the player to complete a simple task in order to proceed (e.g. opening the door requires pulling a lever of a certain color). There is no time limit for completing each task, but for the whole game there is a limit of 5 minutes. The game was constructed with BubbelWorld, a 3D game engine created by computer science students of Lublin University of Technology, not yet available publicly. The application interface is presented in Figure 5.

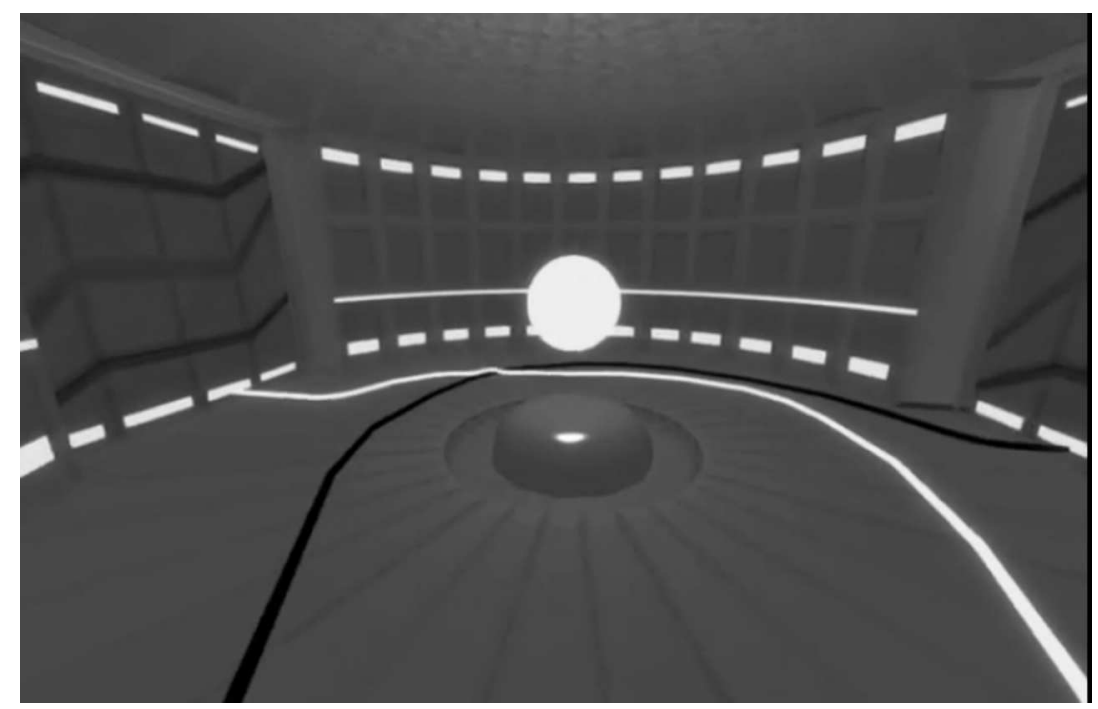

Figure 5. Interface of the Colorblind Maze game (source: own work) 


\section{Results}

The results of both previously mentioned experiments are presented in Tables 2 and 3 .

Table 2. Results for experiment 1

\begin{tabular}{|c|c|c|c|c|c|c|}
\hline \multirow{2}{*}{$\begin{array}{l}\text { system } \\
\text { diagnosis }\end{array}$} & \multirow{2}{*}{$\begin{array}{c}\text { medical } \\
\text { diagnosis }\end{array}$} & \multirow{2}{*}{$\begin{array}{l}\text { diagnosed } \\
\text { persons }\end{array}$} & \multicolumn{4}{|c|}{ diagnosis results } \\
\hline & & & TP & $\mathrm{FP}$ & $\mathrm{TN}$ & $\mathrm{FN}$ \\
\hline \multicolumn{7}{|c|}{ colorblind group } \\
\hline \multirow{2}{*}{ protanopia } & protanopia & 1 & 1 & 0 & 0 & 0 \\
\hline & dichromacy & 4 & 4 & 0 & 0 & 0 \\
\hline protanomaly & $\begin{array}{l}\text { anomalous } \\
\text { trichromacy }\end{array}$ & 1 & 1 & 0 & 0 & 0 \\
\hline deuteranopia & dichromacy & 4 & 4 & 0 & 0 & 0 \\
\hline tritanomaly & $\begin{array}{l}\text { anomalous } \\
\text { trichromacy }\end{array}$ & 2 & 2 & 0 & 0 & 0 \\
\hline \multicolumn{2}{|c|}{ summary } & 12 & 12 & 0 & 0 & 0 \\
\hline \multicolumn{7}{|c|}{ control group } \\
\hline proper color vision & proper color vision & 7 & 7 & 0 & 0 & 0 \\
\hline protanomaly & proper color vision & 2 & 0 & 2 & 0 & 0 \\
\hline deuteranopia & proper color vision & 1 & 0 & 1 & 0 & 0 \\
\hline deuteranomaly & proper color vision & 1 & 0 & 1 & 0 & 0 \\
\hline tritanomaly & proper color vision & 1 & 0 & 1 & 0 & 0 \\
\hline \multicolumn{2}{|c|}{ summary } & 12 & 7 & 5 & 0 & 0 \\
\hline
\end{tabular}

Table 3. Results for experiment 2

\begin{tabular}{|c|c|c|c|c|c|c|}
\hline \multirow{2}{*}{$\begin{array}{l}\text { system } \\
\text { diagnosis }\end{array}$} & \multirow{2}{*}{$\begin{array}{l}\text { medical } \\
\text { diagnosis }\end{array}$} & \multirow{2}{*}{$\begin{array}{l}\text { diagnosed } \\
\text { persons }\end{array}$} & \multicolumn{4}{|c|}{ number of } \\
\hline & & & $\mathrm{TP}$ & $\mathrm{FP}$ & $\mathrm{TN}$ & $\mathrm{FN}$ \\
\hline \multicolumn{7}{|c|}{ colorblind group } \\
\hline \multirow{2}{*}{ protanopia } & protanopia & 1 & 1 & 0 & 0 & 0 \\
\hline & dichromacy & 4 & 4 & 0 & 0 & 0 \\
\hline protanomaly & $\begin{array}{l}\text { anomalous } \\
\text { trichromacy }\end{array}$ & 1 & 1 & 0 & 0 & 0 \\
\hline deuteranopia & dichromacy & 4 & 4 & 0 & 0 & 0 \\
\hline tritanomaly & $\begin{array}{l}\text { anomalous } \\
\text { trichromacy }\end{array}$ & 2 & 2 & 0 & 0 & 0 \\
\hline \multicolumn{2}{|c|}{ summary } & 12 & 12 & 0 & 0 & 0 \\
\hline \multicolumn{7}{|c|}{ control group } \\
\hline proper color vision & proper color vision & 9 & 9 & 0 & 0 & 0 \\
\hline protanomaly & proper color vision & 1 & 0 & 1 & 0 & 0 \\
\hline deuteranopia & proper color vision & 1 & 0 & 1 & 0 & 0 \\
\hline tritanomaly & proper color vision & 1 & 0 & 1 & 0 & 0 \\
\hline \multicolumn{2}{|c|}{ summary } & 12 & 9 & 3 & 0 & 0 \\
\hline
\end{tabular}




\section{Research Analysis and Conclusions}

The analysis of all the experiments based on the proposed interactive method for detecting colorblindness show that it is an interesting alternative to currently used methods, especially considering screening tests.

The first experiment proved that individual features, such as agility or reflex, have a negative influence on the diagnostic process, as - due to a higher number of errors - the system classified almost $2 / 3$ more interviewees as potentially colorblind (in comparison to the second experiment). However, it must be noted that all confirmed cases of colorblindness were detected and diagnosed properly. Each "false positive" was later excluded by expert evaluation.

On the other hand, switching from a 2D to 3D environment had almost no impact on the diagnostic process - the results of the Colorblind Maze experiment neared the results of the "select a square" game. The amount of time needed for developing a 3D game and the computing power needed to run it, however, disputes the necessity and profitability of such a solution. Moreover, the game-based method is designed to be used mainly for screening tests - making quick diagnosis a priority. Considering this, reserving more than 5 minutes per tested person for completion of the game is simply unacceptable.

Although it was proven that all of the proposed games may be used

for colorblindness detection, the most optimal game - in terms of speed, simplicity and relative low random error rate - turned out to be the simplest one, "select a square". This is actually an advantage, as its technical requirements are low and the rules are simple - features which should allow it to reach a wider audience.

\section{R E F E R E N C E S}

Bober, D., Laskowski, M., \& Kęsik, J. (2011). Interactive method of detecting color vision disorders in candidates for drivers. Studies \& Proceedings of Polish Association for Knowledge Management, 42, 5-15.

Campbell, J. L., Spalding, J. A. B., \& Mir, F. A. (2004). The description of physical signs of illness in photographs by physicians with abnormal colour vision. Clinical and Experimental Optometry, 87(4-5), 334-338.

Colourblindawarenessorg. (2015). 1 in every classroom. Retrieved 1 June, 2015, from http://www.colourblindawareness.org/about-us/1ineveryclassroom/

DeMarco, P., Pokorny, J., \& Smith, V. C. (1992). Full-spectrum cone sensitivity functions for X-chromosome-linked anomalous trichromats. Journal of the Optical Society of America A, 9, 1465-1476. 
Derefeldt, G., Hedin, C. E., \& Skoog, K. O. (1995). Colour Vision Deficiencies: Matching and Confusion of Computer Colours. DIANE Publishing.

French, A. L., Rose, K., Thompson, K., \& Cornell, E. (2008). The evolution of colour vision testing. Australian Orthoptic Journal, 40(2), 7-15.

Laskowski, M. (2015). Automation of colorblindness detection and problem of error threshold in computer-based screening test. Communications in Computer and Information Science, 538, 45-56.

Machado, G. M., Oliveira, M. M., \& Fernandes, L. A. F. (2009). A Physiologicallybased Model for Simulation of Color Vision Deficiency. IEEE transactions on visualization and computer graphics, 15(6), 1291-1298.

Masataka, O., \& Kei, I. (2008). Color Universal Design (CUD) - How to make figures and presentations that are friendly to Colorblind people. Japan: Jikei Medical School.

McIntyre, D. (2002). Colour Blindness: Causes and Effects. Chester: Dalton Publishing.

Mushaboomdesigncom. (2015). Digital Printing. Retrieved 21 June, 2015, from http://www.mushaboomdesign.com/Pages/technical/05_06_digitalprinting. html

Opticaldiagnosticscom. (2015). Color Vision Defects. Retrieved 20 June, 2015, from http://www.opticaldiagnostics.com/info/color_vision_defects.html

Shevell, S. K. (2003). The Science of Color (2nd Ed.). Oxford, UK: Elsevier.

Spalding, J. A. B. (2004). Confessions of a colour blind physician. Clinical and Experimental Optometry, 87(4-5), 344-349.

Steward, J. M., \& Cole, B. L. (1989). What do color vision defectives say about everyday tasks? Optometry and Vision Science, 66(5), 288-295.

Yates, J. T., \& Heikens, M. F. (2001). Colour Vision Testing - Methodologies: Update and Review. In J. P. Menu, \& D. Ivan (Eds.) RTO technical report 16. Operational Colour Vision in the Modern Aviation Environment. Neuillysur-Seine, France: RTO, NATO. 